\title{
Search for pulsars and transients with the GMRT
}

\author{
Bhaswati Bhattacharyya ${ }^{1}$ \\ ${ }^{1}$ NCRA-TIFR, \\ Pune University Campus, Ganeshkhind, Pune - 411007 \\ email: bhaswati@ncra.tifr.res.in
}

\begin{abstract}
This paper details on the discovery of 21 pulsars using the Giant Metrewave Radio Telescope (GMRT) from targeted (Fermi directed search) and blind surveys (GMRT High Resolution Southern Sky - GHRSS) and results from the follow up studies. We discovered seven millisecond pulsars (MSPs) in the Fermi directed searches, which are the first Galactic MSPs discovered with the GMRT. We have discovered 13 pulsars (including a MSP and two mildly recycled pulsars) with the GHRSS survey, which is an off-Galactic-plane survey at $322 \mathrm{MHz}$ with complementary target sky (declination range -40 deg to $-54 \mathrm{deg}$ ) to other ongoing lowfrequency surveys by GBT and LOFAR. The simultaneous time-domain and imaging study for localising pulsars and transients and efficient candidate investigation with machine learning are some of the features of the GHRSS survey, which are also finding application in the SKA design methodology.
\end{abstract}

Keywords. (stars:) pulsars: general, (stars:) pulsars: individual (J1544+4947, J1227-4853), instrumentation: interferometers, techniques: miscellaneous, surveys, gamma rays: observations

\section{Introduction}

Pulsars still have tremendous untapped potential to probe the behaviour of matter, energy, space and time under extra-ordinarily diverse conditions. Investigation of single pulse behaviour of normal pulsars having spin period $>30 \mathrm{~ms}$ can exhibit interesting individual properties, like glitches, profile state changes, nulling and intermittency. Stability, compactness second only to black holes, and their presence in binary systems, makes millisecond pulsars (MSPs) ideal laboratories to test the physics of gravity and as detectors for long-wavelength gravitational waves (GWs). Millisecond pulsars (MSPs) are still a small population compared to the normal pulsars and much diversity in their intrinsic characteristics as well as evolutionary history are yet to be explored. Even though pulsars are frequently getting discovered with ongoing surveys at major telescopes over the world, presently known population is only $1 \%$ of prediction. When blindly searched in the radio band, the MSPs are difficult targets due their intrinsic faint nature, and their detection generally requires long exposures even with large radio telescopes. So targeted searches with apriori knowledge of possible position is a effective way to search for MSPs (Ferrara et al. (2017)). We have carried out Fermi-directed search for MSPs with the GMRT, as a part of Fermi pulsar search consortium.

However, since one does not have apriori information about position of majority of the pulsars sensitive blind surveys are being designed to detect those. We are conducting the GMRT High Resolution Southern Sky (GHRSS) survey for pulsars and transients with the GMRT, targeting a portion of sky complementary to other ongoing low-frequency surveys. 

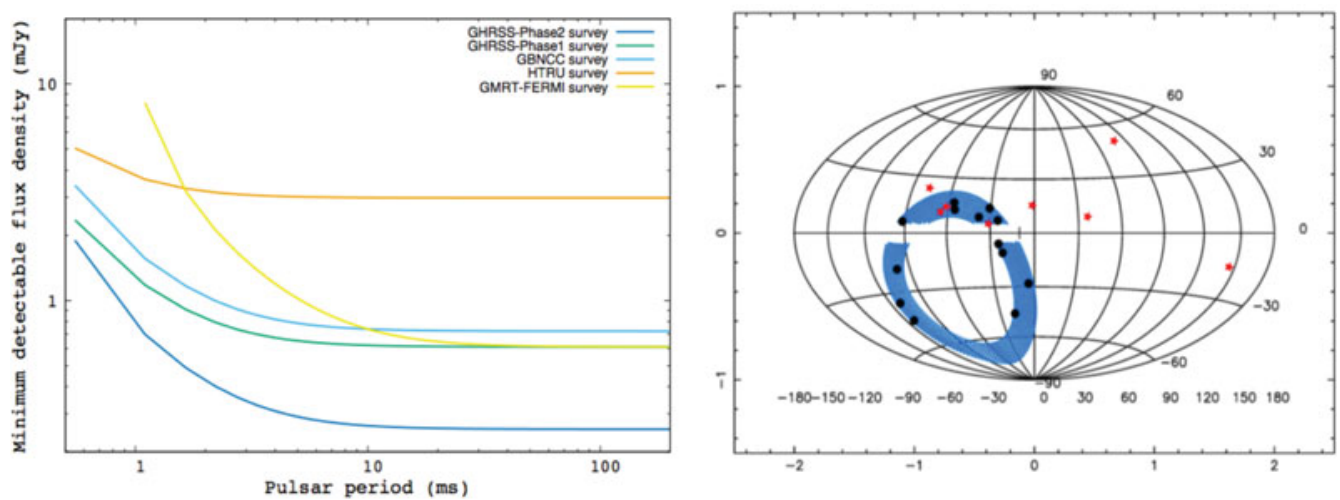

Figure 1. Left panel: Estimated sensitivity of the Fermi-directed search with the GMRT, GHRSS survey Phase 1, GBNCC and the HTRU survey. Right panel: Pulsars discovered by GMRT between 2012-2017 from targeted and blind surveys. Fermi-directed discoveries - stars, GHRSS discoveries - solid circles, sky coverage of GHRSS survey - shaded region.

In addition to the regular emission from pulsars, highly dispersed fast radio bursts (FRBs) of extragalactic origin are also detected in time domain surveys. Moreover, lowfrequency detection of FRBs is important considering the fact that no FRB is detected below $800 \mathrm{MHz}$.

\section{Time domain study with GMRT}

The GMRT is a multi-element aperture synthesis telescope consisting of 30 antennas, each of $45 \mathrm{~m}$ diameter, spread over a region of $25 \mathrm{~km}$ diameter and operating at 5 different wave bands from $150 \mathrm{MHz}$ to $1450 \mathrm{MHz}$. The GMRT Software Backend, built using COTS components, is a fully real-time backend that supports a FX correlator and a beamformer for an array of 32 dual polarized signals, Nyquist sampled at 33 or $66 \mathrm{MHz}$ (Roy et al. (2010)). Time domain study with the GMRT is aided by it's sensitivity and simultaneous imaging capability.

\subsection{Sensitivity}

As pulsars have steep radio spectra the GMRT is ideal for searches away from the Galactic plane. Right panel of Figure 1 plots the estimated sensitivity calculated with the radiometer equation for the Fermi directed survey at GMRT, GHRSS survey Phase-1 (with $32 \mathrm{MHz}$ bandwidth), the GBNCC at GBT and the HTRU at Parkes. The sensitivity estimations are at $322 \mathrm{MHz}$ for $5-\sigma$ detection with $10 \%$ duty cycle in cold sky. The flux densities are scaled using -1.4 spectral index to derive the corresponding $322 \mathrm{MHz}$ values. For Fermi-directed survey with 512 frequency channel over $32 \mathrm{MHz}$ observing band (frequency resolution $\sim 0.062 \mathrm{MHz}$, time resolution $\sim 61$ microsecond) at $322 \mathrm{MHz}$ we calculate $5-\sigma$ detection sensitivity of $0.9 \mathrm{mJy}$ for $30 \mathrm{~min}$ pointing. Sensitivity is improved by $\sim 2.5$ times with enhanced time and frequency resolution modes $(0.015 \mathrm{MHz}$, 61 microsecond) developed for the GHRSS survey. Calculated theoretical sensitivity for 10 mins pointing duration for the GHRSS at $322 \mathrm{MHz}$ is $0.5 \mathrm{mJy}$ for $32 \mathrm{MHz}$ observing band-width.

\subsection{Image Domain Localisation}

Discovery position of pulsars and transients are subject to large uncertainty of the observing beam (e.g. $\pm 40^{\prime}$ for GMRT-322, $\pm 18^{\prime}$ for GBT-350, $\pm 7^{\prime}$ for Parkes-Lband) 
hinder sensitive follow up studies of these newly discovered objects using coherent beams of array telescopes, or at higher frequencies using single dishes. We can localise the newly discovered pulsars and transients in the image plane with the GMRT interferometric array with an accuracy of better than $\pm 10^{\prime \prime}$ (half of the typical synthesized beam used in the image made at $322 \mathrm{MHz}$ ) using gated imaging of pulsars (Roy et al. (2013)).

\section{Fermi directed search for MSPs with the GMRT}

Using the GMRT we performed deep observations to search for radio pulsations in the directions of unidentified Fermi Large Area Telescope (LAT) $\gamma$-ray sources. With the aid of reduced quantization noise and high time resolution survey mode, the GMRT software backend (GSB) enhanced the search sensitivity for nearby MSPs by $\sim 80 \%$ than what was previously possible. The capability of the GMRT in finding MSPs, even in tight binaries, is demonstrated by the discovery of seven MSPs in Fermi directed searches in mid and high Galactic latitudes (Bhattacharyya et al. (2013)). These include the first Galactic field MSPs discovered at the GMRT. Two of the discoveries are serendipitous in-beam MSPs at a large offset from the search centers, even outside the half power beam width. Some of the objects that the GMRT has discovered, are already pushing the boundaries of the known parameter space of MSPs; for example, J1544+4947 - eclipsing BlackWidow MSP (Bhattacharyya et al. (2013)) and J1536-4948 - a MSP having very wide pulse profile (possibly widest among MSPs). We have also discovered J1227-4853 - a transitional MSP caught in the act of transition from a Low-mass X-ray Binary to radio MSP. Parameters of these MSPs are listed in Table 1. While folding data with radio timing model, LAT pulsation is detected from 4 of the MSPs discovered by the GMRT. Since two of the MSPs are unassociated with any $\gamma$-ray source we do not expect to detect LAT pulsation from these. We have not yet detected LAT pulsation from the remaining MSP, indicating that possibly the pulsar is not associated with the $\gamma$-ray source.

\section{GMRT high resolution southern sky survey}

We are conducting the GHRSS survey $\dagger$ from mid 2013. Till now we covered $\sim 1800$ square degree of sky (62\% of the total target sky). Survey description and initial discovery of 10 pulsars are reported in Bhattacharyya et al. (2016). Left panel of Figure 1 showes the GHRSS target sky and the pulsars discovered in this survey. Till now we have discovered 13 pulsars (listed in Table 1) from this survey including one MSP, one pulsar with LAT pulsation and two mildly recycled pulsars. So our present discovery rate is 13 pulsars in $1800 \mathrm{deg}^{2}$, i.e. 0.007 pulsars per $\mathrm{deg}^{2}$, which is one of the highest among off-galactic plane surveys. Following we provide description of some of the pulsars discovered with the GHRSS survey.

- We have discovered $\gamma$-ray pulsations aided by the radio timing from the GMRT, of our discovery PSR J0514-4408. This pulsar has a double peaked profile at $322 \mathrm{MHz}$ which evolves to a single peaked $\gamma$-ray profile. The relative alignment of the $\gamma$-ray and radio peaks is unusual for a young, non-recycled pulsar.

- PSR J2144-5237 is a MSP with a multi-component profile having significant evolution with frequency.

- PSR J1516-43 is a mildly recycled pulsar in a 223 days orbit with a companion mass of $0.4 \mathrm{M}_{\odot}$.

$\dagger$ http://www.ncra.tifr.res.in/ncra/research/research-at-ncra-tifr/research-areas/ pulsarSurveys/GHRSS 
Table 1. Parameters of the pulsars discovered with the GMRT between 2012-2017 with targeted and blind search.

\begin{tabular}{|c|c|c|c|c|}
\hline Pulsar name & $\begin{array}{l}\text { Period } \\
(\mathrm{ms})\end{array}$ & $\begin{array}{l}\text { Dispersion measure } \\
\quad\left(\mathrm{pc} \mathrm{cm}^{-3}\right)\end{array}$ & $\begin{array}{c}\text { Type of pulsar/Orbital period } \\
\text { (day) }\end{array}$ & $\left|\underset{(\mathrm{m} J y)}{\text { Flux })^{\text {density }}{ }^{\ddagger}}\right|$ \\
\hline PSR J0248+42 & 2.60 & 48.2 & isolated MSP & 1.9 \\
\hline PSR J0418-4154 ${ }^{\dagger}$ & 757.11 & 24.5 & normal PSR & 10.3 \\
\hline PSR J0514-4408 ${ }^{\dagger}$ & 302.2 & 15.4 & normal PSR & 9.7 \\
\hline PSR J0702-4956 ${ }^{\dagger}$ & 666.66 & 98.7 & normal PSR & 15.7 \\
\hline PSR J0919-42 ${ }^{\dagger}$ & 812.6 & 57.8 & normal PSR & 6.4 \\
\hline PSR J1120-3618 & 5.55 & 45.1 & MSP & 0.3 \\
\hline PSR J1207-5050 & 4.84 & 50.6 & isolated MSP & 0.5 \\
\hline PSR J1227-4853 & 1.686 & 43.4 & 0.28 & 6.6 \\
\hline PSR J1239-48 & 653.89 & 107.6 & normal PSR & 0.4 \\
\hline PSR J1255-46 ${ }^{\dagger}$ & 52.0 & 42.9 & mildly recycled & 0.8 \\
\hline PSR J1456-48 & 536.81 & 133.0 & 15 & 1.2 \\
\hline PSR J1516-43 & 36.03 & 70.2 & mildly recycled & 0.7 \\
\hline PSR J1536-4948 & 3.08 & 38.0 & 62.5 & 12 \\
\hline PSR J1544+4937 & 2.16 & 23.2 & 0.12 & 2.6 \\
\hline PSR J1559-44 ${ }^{\dagger}$ & 1169.89 & 122.0 & normal PSR & 1.7 \\
\hline PSR J1646-2142 & 5.85 & 29.7 & isolated MSP & 0.7 \\
\hline PSR J1708-52 ${ }^{\dagger}$ & 449.62 & 102.6 & normal PSR & 1.4 \\
\hline PSR J1726-52 ${ }^{\dagger}$ & 631.84 & 119.7 & normal PSR & 0.7 \\
\hline PSR J1828+0625 & 3.63 & 22.4 & MSP & 1.0 \\
\hline PSR J1947-43 ${ }^{\dagger}$ & 180.94 & 29.9 & normal PSR & 4.7 \\
\hline PSR J2144-5237 ${ }^{\dagger}$ & 5.04 & 19.0 & 10.58 & 1.6 \\
\hline
\end{tabular}

Notes: ${ }^{\dagger}$ : GHRSS discoveries, $\stackrel{\ddagger}{\ddagger}$ Flux density is without primary beam correction.

- PSR J1255-46 is another mildly recycled pulsar.

We demonstrated rapid convergence in pulsar timing with a more precise position obtained with imaging and achieved long-term timing solutions for GHRSS pulsars. In addition to periodicity searches, the single pulse searches of the GHRSS survey can reveal sources like Rotating Radio Transients (RRATs) and FRBs. The full survey is expected to detect $\sim 4$ fast radio bursts at fluence of $3 \mathrm{Jy}$-ms assuming a flat spectra. Even non-detection of FRBs in $50 \%$ of the survey yields a 2-sigma upper limit of 2000 events sky $^{-1}$ day $^{-1}$ at $322 \mathrm{MHz}$, which can put a constraining upper-limit on FRB spectral index. Aided by the upgraded GMRT with increased sensitivity, we plan to complete the remaining part of the GHRSS survey with the wider bandwidth (Roy (2017)).

\section{References}

Ferrara, A. et al. 2017, Pulsar Astrophysics - The Next 50 Years Proceedings of IAU Symposium. Bhattacharyya B. et al. 2013, ApJ Letters, 772, 12.

Bhattacharyya B. et al. 2016, ApJ Letters, 817, 130.

Roy, J. 2017, Pulsar Astrophysics - The Next 50 Years Proceedings of IAU Symposium.

Roy, J. et al. 2013, ApJ Letters, 765, 45.

Roy, J. et al. 2010, Experimental Astronomy, 28, 55. 